\title{
CARACTERISTICAS, OPERACIONALIDADE E PRODUÇÃO DA FROTA DE CAMARONEIROS DO ESTADO DO RIO DE JANEIRO (BRASIL)
}

\author{
Characteristics, operation and production of the shrimp trawler \\ fleet in Rio de Janeiro State (Brazil)
}

Melquíades Pinto Paiva ${ }^{1,3}$, Gualdino Afonso Té ${ }^{1}$, Antônio Alberto da Silveira Menezes²

\section{RESUMO}

Este trabalho trata das características, operacionalidade e produção da frota industrial de camaroneiros do estado do Rio de Janeiro (Brasil), nos anos de 1993 - 1997. As pescarias são dirigidas para as capturas do camarão-rosa = Farfantepenaeus brasiliensis (Latreille, 1817) + Farfantepenaeus paulensis (Pérez Farfante, 1967). A frota é constituída por barcos de aço e de madeira, com grande variação da maior parte de suas características, tendo idade avançada e pouca renovação. As pescarias duram 8 - 9 meses por ano, por causa do defeso, sendo mais importantes nos meses de julho-outubro. A variação do tempo anual de operação dos barcos se reflete nas capturas do camarãorosa, que alcança média pouco superior a 3,8 t/ano, quando os barcos realizam $181-210$ dias de pesca/ano. A tendência decrescente da produção do camarão-rosa, nos sucessivos anos, decorre da queda de produtividade das pescarias.

Palavras-chaves: camarão-rosa, frota camaroneira, pescarias industriais, Rio de Janeiro (Brasil).

\section{ABSTRACT}

This paper deals with the characteristics, operation and production of the industrial shrimp-trawler fleet in the State of Rio de Janeiro (Brazil) in $1993-1997$ years. The fisheries are directed to catches of the pink-shrimp = Farfantepenaeus brasiliensis (Latreille, 1817) + Farfantepenaeus paulensis (Pérez Farfante, 1967). The fleet is composed by steel and wooden boats, with large variation in the majority of their characteristics, very old in age, having little renovation. The fisheries cover 8 - 9 months/year, due to the closed season, with major activities in July - October. The annual time variation in the shrimp-trawler operations is reflected in the pink-shrimp catches, that reach a little more of 3,8 t/year, when the boats made $181-210$ fishing days/year. There is a decreasing trend in pink-shrimp production along the years, due to the fall in fishery productivity.

Key words: pink-shrimp, shrimp-trawler fleet, industrial fisheries, Rio de Janeiro (Brazil).

\footnotetext{
${ }^{1}$ Departamento de Biologia Marinha / Instituto de Biologia / Universidade Federal do Rio de Janeiro / Cidade Universitária - Ilha do Fundão / 21944-970 Rio de Janeiro - RJ - Brasil.

${ }^{2}$ Instituto Brasileiro do Meio Ambiente e dos Recursos Naturais Renováveis / Superintendência Estadual do Rio de Janeiro / Praça XV de Novembro, 42/3․ andar / 20010-010 Rio de Janeiro - RJ - Brasil.

${ }^{3}$ Bolsista do Conselho Nacional de Desenvolvimento Científico e Tecnológico (CNPq).
} 


\section{INTRODUÇÃO}

A explotação industrial camaroneira, ao longo da costa sudeste/sul do Brasil, é dirigida para a captura do camarão-rosa = Farfantepenaeus brasiliensis (Latreille, 1817) + Farfantepenaeus paulensis (Pérez Farfante, 1967); as pescarias ocorrem desde o estado do Espírito Santo, em frente à foz do rio Doce, até o estuário da lagoa dos Patos, no estado do Rio Grande doSul, em profundidades de $30-100 \mathrm{~m}$ e em fundos de areia e lama, com safra nos meses de setembro a dezembro (Paiva, 1997).

As pescarias industriais do camarão-rosa, em águas costeiras do estado do Rio de Janeiro, têm relevante importância, com frota baseada em seus portos. Os desembarques são concentrados na ilha da Conceição (Niterói), Pedra de Guaratiba (Rio de Janeiro) e Angra dos Reis. É praticada por camaroneiros, em sua grande maioria operando com redes duplas (doublerig) - (Figura 1), embora pequena porcentagem de barcos utilizam o arrasto simples de popa, atuando em pesqueiros mais próximos da costa.

As estatísticas pesqueiras, disponíveis a partir de 1964 e recentes trabalhos publicados (Paiva, 1997; Costa et al. 1998), comprovam tendência decrescente da produção industrial do camarão-rosa, nos desembarques em portos do estado do Rio de Janeiro

O presente trabalho trata das características, operacionalidade e produção da frota de camaroneiros do estado do Rio de Janeiro (Brasil).

\section{MATERIAL E MÉTODOS}

Os dados que suportam este trabalho são oriundos do Instituto Brasileiro do Meio Ambiente e dos Recursos Naturais Renováveis / Superintendência Estadual do Rio de Janeiro (IBAMA/ SUPES - RJ), encontrados em dois conjuntos distintos de documentos: registros das embarcações - com a identificação da embarcação e do seu proprietário ou arrendatário, tipo de pesca e equipamentos existentes; mapas-de-bordo dos camaroneiros - com a identificação da embarcação, saída e chegada do/no porto, esforço de pesca e captura do camarão-rosa.

No tocante aos registros dos camaroneiros, consideramos as embarcações cadastradas, agrupadas em dois tipos, segundo a construção: barcos de madeira e barcos de aço. Levamos em conta 19 características destes barcos, com os correspondentes parâmetros de variação (Tabela I). Agrupamos as idades dos barcos por classes e tipos, possibilitando estimativas das idades médias em 1997, inclusive para o total das unidades registradas (Tabela II), que é inferior ao do total de camaroneiros em operação nos anos considerados, com exceção para 1997.

Procuramos conhecer as freqüências relativas dos camaroneiros em operação, segundo os números de meses e a distribuição nos meses dos diferentes anos, para avaliação dos padrões temporais de operacionalidade da frota (Tabelas III - IV).

De cada camaroneiro que operou no período de 1993 - 1997, com base em portos fluminenses, foram anotados os totais anuais dos dias de pesca, lances, horas de arrasto e produção do camarão-rosa. Depois estes dados foram calculados para o conjunto dos anos considerados, no total de 540 camaroneiros/ano (Tabela V).

A conservação do camarão-rosa vem sendo feita com gelo em barra ou escama, nos camaroneiros menores e/ou mais velhos. Nos outros, encontram-se congelador e câmara frigorífica.

Figura 1 - Camaroneiro Universo II, de casco de aço, fundeado em Ponta d' Areia (Niterói - RJ - Brasil ). Foto cedida pelo armador Francisco Bremen Kamp (Funelli). 
Tabela I - Características de parte da frota industrial de camaroneiros, baseados em portos do estado do Rio de Janeiro (Brasil), que operaram nos anos de 1993 - 1997.

\begin{tabular}{|c|c|c|c|c|c|c|}
\hline \multirow{2}{*}{ Característica } & \multirow{2}{*}{$\begin{array}{l}\text { Registros } \\
\qquad\left(\mathrm{n}^{\circ}\right)\end{array}$} & \multicolumn{2}{|c|}{ Amplitude } & \multirow{2}{*}{$\frac{\text { Média }}{(x)}$} & \multirow{2}{*}{$\begin{array}{l}\text { Desvio } \\
\text { padrão } \\
\text { (s) }\end{array}$} & \multirow{2}{*}{$\begin{array}{l}\text { Coeficiente de } \\
\text { variação } \\
(\%)\end{array}$} \\
\hline & & mínimo & máximo & & & \\
\hline \multicolumn{7}{|l|}{ Barcos de aço $(n=13)$} \\
\hline comprimento total (m) & 13 & 19,14 & 33,75 & 21,37 & 3,83 & 17,92 \\
\hline boca moldada $(\mathrm{m})$ & 13 & 5,07 & 7,08 & 5,97 & 0,54 & 9,04 \\
\hline pontal (m) & 13 & 2,61 & 3,80 & 3,17 & 0,32 & 10,09 \\
\hline calado de vante (m) & 10 & 0,40 & 3,20 & 3,36 & 0,95 & 0,28 \\
\hline calado de ré (m) & 9 & 0,80 & 3,60 & 4,90 & 0,81 & 0,17 \\
\hline calado máximo $(\mathrm{m})$ & 9 & 0,80 & 3,60 & 4,90 & 0,81 & 0,17 \\
\hline contorno $(\mathrm{m})$ & 6 & 7,60 & 10,56 & 9,42 & 1,02 & 10,83 \\
\hline arqueação bruta (t) & 13 & 71,86 & 100,91 & 106,34 & 44,88 & 42,20 \\
\hline arqueação líquida (t) & 13 & 24,00 & 106,14 & 45,84 & 26,89 & 58,66 \\
\hline tanque de combustível (l) & 12 & 200,00 & $22.000,00$ & $6.225,00$ & $10.443,13$ & 64,36 \\
\hline tanque de lubrificante (l) & 12 & 20,00 & $2.000,00$ & 825,83 & 884,97 & 107,16 \\
\hline tanque de água doce (l) & 12 & 200,00 & $7.000,00$ & $4.883,33$ & $3.383,60$ & 69,29 \\
\hline total dos tripulantes $\left(\mathrm{n}^{\circ}\right)$ & 10 & 5 & 24 & 12,00 & 7,04 & 58,67 \\
\hline pescadores $\left(n^{\circ}\right)$ & 11 & 3 & 24 & 10,90 & 6,65 & 61,00 \\
\hline autonomia mar (dias) & 9 & 5,00 & 30,00 & 19,11 & 7,47 & 39,09 \\
\hline raio de ação (mi) & 8 & 15,00 & 100,00 & 117,55 & 34,69 & 29,51 \\
\hline potência motor (HP) & 12 & 38,50 & 430,00 & 270,42 & 123,83 & 45,79 \\
\hline rotação motor (RPM) & 11 & $1.500,00$ & $3.600,00$ & 207,73 & 603,47 & 290,51 \\
\hline motor auxiliar (HP) & 8 & 18,00 & 65,00 & 43,87 & 16,20 & 36,93 \\
\hline \multicolumn{7}{|c|}{ Barcos de madeira $(n=66)$} \\
\hline comprimento total (m) & 66 & 15,70 & 34,85 & 19,68 & 2,76 & 14,02 \\
\hline boca moldada (m) & 65 & 4,30 & 9,70 & 5,18 & 0,81 & 15,64 \\
\hline pontal (m) & 66 & 1,80 & 3,50 & 2,35 & 0,35 & 14,89 \\
\hline calado de vante (m) & 60 & 0,40 & 2,90 & 1,09 & 0,59 & 54,13 \\
\hline calado de ré (m) & 61 & 0,80 & 3,20 & 1,63 & 0,45 & 27,61 \\
\hline calado máximo (m) & 59 & 0,80 & 3,30 & 1,52 & 0,47 & 30,92 \\
\hline contorno $(\mathrm{m})$ & 47 & 4,50 & 13,00 & 6,37 & 2,01 & 31,55 \\
\hline arqueação bruta (t) & 66 & 26,20 & 193,45 & 46,04 & 24,02 & 52,17 \\
\hline arqueação líquida (t) & 66 & 12,96 & 154,40 & 24,57 & 19,99 & 81,36 \\
\hline tanque de combustível (l) & 59 & 50,00 & $22.000,00$ & $9.487,97$ & $7.952,47$ & 83,82 \\
\hline tanque de lubrificante (l) & 61 & 5,00 & $2.000,00$ & 200,36 & 404,70 & 201,99 \\
\hline tanque de água doce (l) & 62 & 60,00 & $10.000,00$ & $2.790,64$ & $2.168,74$ & 77,71 \\
\hline total dos tripulantes $\left(\mathrm{n}^{\circ}\right)$ & 61 & 5 & 23 & 8,77 & 4,19 & 47,78 \\
\hline pescadores $\left(n^{\circ}\right)$ & 61 & 5 & 23 & 8,29 & 4,05 & 48,89 \\
\hline autonomia de mar (dias) & 60 & 4,00 & 30,00 & 14,12 & 3,82 & 27,05 \\
\hline raio de ação (mi) & 58 & 1,50 & 100,00 & 28,85 & 28,10 & 97,40 \\
\hline potência motor (HP) & 62 & 90,00 & 900,00 & 271,16 & 105,86 & 39,04 \\
\hline rotação motor (RPM) & 59 & 320,00 & $4.000,00$ & $1.690,77$ & 647,56 & 38,30 \\
\hline motor auxiliar (HP) & 39 & 6,00 & 120,00 & 63,58 & 46,66 & 73,39 \\
\hline
\end{tabular}

Observação: foram considerados apenas os barcos registrados no IBAMA - Superintendência Estadual do Rio de Janeiro. 
Tabela II - Idades de parte da frota de camaroneiros, baseados em portos do estado do Rio de Janeiro (Brasil), nos anos de 1993 - 1997. Idades referentes ao ano de 1997.

\begin{tabular}{c|c|c|c|c|c|c|c|c|c}
\hline \multirow{2}{*}{$\begin{array}{c}\text { Idades } \\
(\text { anos })\end{array}$} & \multicolumn{3}{|c|}{ Barcos de aço } & \multicolumn{3}{c|}{ Barcos de madeira } & \multicolumn{3}{|c}{ Frota camaroneira } \\
\cline { 2 - 9 } & $\mathrm{n}^{\circ}$ & $\%$ & $\overline{\mathrm{x}}$ & $\mathrm{n}^{\circ}$ & $\%$ & $\overline{\mathrm{x}}$ & $\mathrm{n}^{\circ}$ & $\%$ & $\overline{\mathrm{x}}$ \\
\hline-10 & - & - & - & 2 & 3,0 & 9,0 & 2 & 2,5 & 9,0 \\
$11-15$ & 4 & 30,8 & 12,3 & - & - & - & 4 & 5,0 & 12,3 \\
$16-20$ & 1 & 7,7 & 16,0 & 2 & 3,0 & 17,0 & 3 & 3,7 & 16,7 \\
$21-25$ & 1 & 7,7 & 24,0 & 8 & 11,6 & 23,6 & 9 & 11,1 & 23,7 \\
$26-30$ & 6 & 46,1 & 28,2 & 34 & 49,3 & 27,5 & 40 & 48,1 & 27,6 \\
$31-35$ & - & - & - & 9 & 13,0 & 32,4 & 9 & 11,1 & 32,4 \\
$36-40$ & - & - & - & 1 & 1,4 & 39,0 & 1 & 1,2 & 39,0 \\
$41-45$ & 1 & 7,7 & 42,0 & 3 & 4,3 & 43,7 & 4 & 5,0 & 43,3 \\
$46-50$ & - & - & - & 9 & 13,0 & 48,4 & 9 & 11,1 & 48,4 \\
$51-55$ & - & - & - & 1 & 1,4 & 55,0 & 1 & 1,2 & 55,0 \\
\hline Totais & 13 & 100 & 23,1 & 69 & 100,0 & 30,9 & 82 & 100,0 & 29,6 \\
\hline
\end{tabular}

Observação: para três barcos de madeira dispomos apenas dos dados de idade.

Tabela III - Freqüências relativas dos camaroneiros baseados em portos do estado do Rio de Janeiro (Brasil), por meses de operação, nos anos de 1993 - 1997.

\begin{tabular}{|c|c|c|c|c|c|c|c|}
\hline \multirow{3}{*}{\multicolumn{2}{|c|}{$\begin{array}{c}\text { Meses } \\
\left(\mathrm{n}^{\circ}\right)\end{array}$}} & \multicolumn{6}{|c|}{ Camaroneiros em operação (\%) } \\
\hline & & \multicolumn{5}{|c|}{ anos } & \multirow{2}{*}{ médias } \\
\hline & & 1993 & 1994 & 1995 & 1996 & 1997 & \\
\hline \multicolumn{2}{|c|}{1} & 14,3 & 10,3 & 19,2 & 21,8 & 5,3 & 14,2 \\
\hline \multicolumn{2}{|c|}{2} & 11,2 & 9,4 & 8,0 & 20,2 & 7,9 & 11,4 \\
\hline \multicolumn{2}{|c|}{3} & 13,3 & 6,8 & 6,4 & 7,3 & 11,8 & 9,1 \\
\hline \multicolumn{2}{|c|}{4} & 15,3 & 6,0 & 9,6 & 4,8 & 9,2 & 9,0 \\
\hline \multicolumn{2}{|c|}{5} & 15,3 & 9,4 & 7,2 & 5,6 & 13,2 & 10,1 \\
\hline \multicolumn{2}{|c|}{6} & 19,4 & 3,4 & 7,2 & 4,0 & 13,2 & 9,4 \\
\hline \multicolumn{2}{|c|}{7} & 6,1 & 2,6 & 10,4 & 7,2 & 19,7 & 9,2 \\
\hline \multicolumn{2}{|c|}{8} & 5,1 & 4,3 & 23,2 & 9,7 & 17,1 & 11,9 \\
\hline \multicolumn{2}{|c|}{9} & - & 10,3 & 8,8 & 9,7 & 2,6 & 6,3 \\
\hline \multicolumn{2}{|c|}{10} & - & 17,9 & - & 9,7 & - & 5,5 \\
\hline \multicolumn{2}{|c|}{11} & - & 11,1 & - & - & - & 2,2 \\
\hline \multicolumn{2}{|c|}{12} & - & 8,5 & - & - & - & 1,7 \\
\hline \multirow{2}{*}{ Ano } & $\left(n^{\circ}\right)$ & 98 & 117 & 125 & 124 & 76 & 108 \\
\hline & $(\%)$ & 100,0 & 100,0 & 100,0 & 100,0 & 100,0 & 100,0 \\
\hline
\end{tabular}

Tabela IV - Freqüências relativas de camaroneiros baseados em portos do estado do Rio de Janeiro (Brasil), em operação nos meses dos anos de 1993 - 1997.

\begin{tabular}{|c|c|c|c|c|c|c|c|}
\hline \multirow{3}{*}{\multicolumn{2}{|c|}{ Meses }} & \multicolumn{6}{|c|}{ Camaroneiros em operação (\%) } \\
\hline & & \multicolumn{5}{|c|}{ anos } & \multirow{2}{*}{ médias } \\
\hline & & 1993 & 1994 & 1995 & 1996 & 1997 & \\
\hline \multicolumn{2}{|c|}{ I } & 21,4 & 57,3 & 62,4 & 45,2 & 63,2 & 49,9 \\
\hline \multicolumn{2}{|c|}{ II } & 32,7 & 55,5 & - & 52,4 & 71,1 & 42,3 \\
\hline \multicolumn{2}{|c|}{ III } & - & 50,4 & - & - & - & 10,1 \\
\hline \multicolumn{2}{|c|}{ IV } & - & 47,9 & - & - & - & 9,6 \\
\hline \multicolumn{2}{|c|}{$\mathrm{V}$} & - & 50,4 & 55,2 & 46,0 & 60,5 & 42,4 \\
\hline \multicolumn{2}{|c|}{ VI } & 23,5 & 56,4 & 65,6 & 25,0 & 67,1 & 47,5 \\
\hline \multicolumn{2}{|c|}{ VII } & 50,0 & 66,7 & 62,4 & 48,4 & 67,1 & 58,9 \\
\hline \multicolumn{2}{|c|}{ VIII } & 46,9 & 71,8 & 57,6 & 50,8 & 68,4 & 59,1 \\
\hline \multicolumn{2}{|c|}{ IX } & 41,8 & 60,7 & 37,6 & 50,8 & 68,4 & 51,9 \\
\hline \multicolumn{2}{|c|}{$x$} & 70,4 & 63,2 & 55,2 & 47,6 & 59,2 & 59,1 \\
\hline \multicolumn{2}{|c|}{ XI } & 56,1 & 56,4 & 56,0 & 44,3 & 3,9 & 43,3 \\
\hline \multicolumn{2}{|c|}{ XII } & 71,4 & 39,3 & 55,2 & 55,6 & 3,9 & 45,1 \\
\hline \multirow{2}{*}{ Ano } & $\left(n^{\circ}\right)$ & 98 & 117 & 125 & 124 & 77 & 108 \\
\hline & $(\%)$ & 100,0 & 100,0 & 100,0 & 100,0 & 100,0 & 100,0 \\
\hline
\end{tabular}


Tabela V - Valores médios do esforço de pesca e da produção do camarão-rosa, correspondentes a classes de duração das pescarias da frota camaroneira baseada em portos do estado do Rio de Janeiro (Brasil ), nos anos de 1993 - 1997.

\begin{tabular}{|c|c|c|c|c|c|}
\hline \multirow{2}{*}{$\begin{array}{c}\text { Classes de } \\
\text { tempo } \\
\text { (dias) }\end{array}$} & \multirow{2}{*}{$\begin{array}{c}\text { Barcos em } \\
\text { operação } \\
\left(n^{\circ}\right)\end{array}$} & \multicolumn{3}{|c|}{ Médias do esforço de pesca } & \multirow{2}{*}{$\begin{array}{c}\text { Produçãa } \\
\text { média } \\
(\mathrm{kg})\end{array}$} \\
\hline & & $\begin{array}{l}\text { dias de } \\
\text { pesca }\end{array}$ & $\begin{array}{c}\text { lances } \\
\left(\mathrm{n}^{\circ}\right)\end{array}$ & $\begin{array}{c}\text { horas de } \\
\text { arrasto }\end{array}$ & \\
\hline \multicolumn{6}{|c|}{ Ano: 1993 = 98 camaroneiros } \\
\hline $1-30$ & 33 & 15,6 & 40,2 & 168,4 & 431,6 \\
\hline $31-60$ & 36 & 47,9 & 120,5 & 513,8 & $1.186,0$ \\
\hline $61-90$ & 21 & 72,4 & 190,0 & 781,7 & $1.933,7$ \\
\hline 0 & 6 & 99,5 & 215,0 & 967,3 & $2.130,3$ \\
\hline $121-150$ & 2 & 130,5 & 269,0 & $1.287,0$ & $2.654,0$ \\
\hline \multicolumn{6}{|c|}{ Ano: $1994=117$ camaroneiros } \\
\hline $1-30$ & 27 & 14,5 & 48,9 & 201,1 & 535,7 \\
\hline $31-60$ & 20 & 46,5 & 137,3 & 532,9 & $1.586,4$ \\
\hline $61-90$ & 8 & 72,3 & 197,1 & 778,3 & $1.820,1$ \\
\hline $91-120$ & 14 & 112,8 & 372,0 & $1.400,8$ & $2.904,6$ \\
\hline $121-150$ & 19 & 136,5 & 425,7 & $1.661,8$ & $3.572,3$ \\
\hline $151-180$ & 19 & 160,2 & 486,7 & $1.845,8$ & $3.772,7$ \\
\hline & 9 & 192,9 & 506,3 & $2.074,9$ & $3.875,7$ \\
\hline & - & - & - & - & - \\
\hline $241-270$ & 1 & 248,0 & 922,0 & $3.692,0$ & $3.229,0$ \\
\hline \multicolumn{6}{|c|}{ Ano: $1995=125$ camaroneiros } \\
\hline $1-30$ & 35 & 13,7 & 37,7 & 150,7 & 522,4 \\
\hline & 20 & 45,9 & 118,2 & 474,3 & 918,5 \\
\hline $61-90$ & 24 & 78,1 & 205,1 & 840,4 & $2.076,3$ \\
\hline $91-120$ & 23 & 102,7 & 278,0 & $1.112,5$ & $2.395,3$ \\
\hline $121-150$ & 19 & 130,5 & 347,1 & $1.386,3$ & $3.094,6$ \\
\hline $151-180$ & 3 & 169,0 & 457,0 & $2.066,3$ & $3.353,7$ \\
\hline $181-210$ & 1 & 193,0 & 607,0 & $2.349,0$ & $5.547,0$ \\
\hline
\end{tabular}

\section{Ano : $1996=124$ camaroneiros}

\begin{tabular}{c|c|c|c|c|c} 
Ano : $1996=124$ & camaroneiros \\
$1-30$ & 49 & 14,3 & 44,6 & 180,4 & 306,4 \\
$31-60$ & 20 & 42,1 & 136,4 & 540,5 & 884,7 \\
$61-90$ & 12 & 76,2 & 244,7 & 959,0 & $1.627,6$ \\
$91-120$ & 12 & 107,4 & 341,2 & $1.378,5$ & $2.002,2$ \\
$121-150$ & 13 & 138,6 & 467,6 & $1.839,8$ & $2.538,1$ \\
$151-180$ & 15 & 163,4 & 540,5 & $2.155,3$ & $3.058,5$ \\
$181-210$ & 2 & 186,0 & 514,0 & $2.336,0$ & $2.572,0$ \\
$211-240$ & - & - & - & - & - \\
$241-270$ & 1 & 270,0 & $1.137,0$ & $4.558,0$ & $4.088,0$
\end{tabular}

\section{Ano: $1997=76$ camaroneiros}

\begin{tabular}{c|c|c|c|c|r}
\hline Ano: $1997=76$ camaroneiros \\
\hline $1-30$ & 13 & 19,2 & 80,5 & 359,9 & 343,6 \\
$31-60$ & 17 & 47,2 & 188,4 & 926,2 & 791,4 \\
$61-90$ & 19 & 74,9 & 277,1 & $1.185,3$ & $1.584,2$ \\
$91-120$ & 16 & 104,4 & 437,9 & $1.829,1$ & $1.780,1$ \\
$121-150$ & 8 & 133,3 & 480,5 & $2.036,3$ & $3.039,1$ \\
$151-180$ & 2 & 152,5 & 753,0 & $2.834,0$ & $3.278,0$ \\
$181-210$ & 1 & 182,0 & 693,0 & $2.914,0$ & $4.173,0$ \\
\hline Anos: $1993-1997=540$ camaroneiros/ano \\
\hline $1-30$ & 157 & 14,9 & 45,8 & 189,7 & 423,4 \\
$31-60$ & 113 & 46,2 & 136,1 & 576,9 & $1.185,3$ \\
$61-90$ & 84 & 75,1 & 222,5 & 914,8 & $1.840,8$ \\
$91-120$ & 71 & 105,6 & 337,9 & $1.363,5$ & $2.268,2$ \\
$121-150$ & 61 & 134,5 & 412,2 & $1.650,7$ & $3.103,1$ \\
$151-180$ & 39 & 161,7 & 518,8 & $2.032,5$ & $3.440,4$ \\
$181-210$ & 13 & 200,5 & 592,1 & $2.412,5$ & $3.826,6$ \\
$211-240$ & - & - & - & - & - \\
$241-270$ & 2 & 259,0 & $1.029,5$ & $4.125,0$ & $3.658,5$ \\
\hline
\end{tabular}

Tabela VI - Dados comparativos da produção média anual do camarão-rosa, correspondentes a classes de duração das pescarias dos camaroneiros baseados nos portos do estado do Rio de Janeiro (Brasil), nos anos de 1993 - 1997.

\begin{tabular}{c|r|r|r|r|r|r}
\hline \multirow{2}{*}{$\begin{array}{c}\text { Classes de } \\
\text { tempo }\end{array}$} & \multicolumn{5}{|c}{ Produção do camarão-rosa (\%) } \\
\cline { 2 - 6 } (dias) & 1993 & 1994 & 1995 & 1996 & 1997 & médias \\
\cline { 2 - 6 } & \multicolumn{5}{|c}{ anos } \\
\hline $1-30$ & 101,9 & 126,5 & 123,4 & 72,4 & 81,2 & 100,0 \\
$31-60$ & 100,1 & 133,8 & 77,5 & 74,6 & 66,8 & 100,0 \\
$61-90$ & 105,0 & 98,9 & 112,8 & 88,4 & 86,1 & 100,0 \\
$91-120$ & 93,9 & 128,1 & 105,6 & 88,3 & 78,5 & 100,0 \\
$121-150$ & 85,5 & 115,1 & 99,7 & 81,8 & 97,9 & 100,0 \\
$151-180$ & - & 109,7 & 97,5 & 88,9 & 95,3 & 100,0 \\
$181-210$ & - & 101,3 & 150,0 & 67,2 & 109,1 & 100,0 \\
$211-240$ & - & - & - & - & - & 100,0 \\
$241-270$ & - & 88,3 & - & 111,7 & - & 100,0 \\
\hline Médias & 97,3 & 112,7 & 109,5 & 84,2 & 87,8 & 100,0 \\
\hline
\end{tabular}

\section{RESULTADOS E DISCUSSÃO}

As características mais estáveis dos camaroneiros (coeficientes de variação até $25 \%$ ), em ambos os seus tipos, são as seguintes: barcos de aço - comprimento total, boca moldada, pontal, calados (vante, rée máximo) e contorno; barcos de madeira-comprimento total, boca moldada e pontal. Estas características são as que melhor definem o modelo dos camaroneiros, havendo grande variação das outras levadas em conta (Tabela I).

Os barcos de aço se concentraram em duas faixas, entre $11-15$ e $26-30$ anos, nesta última com pouco menos da metade deles; em média, estavam com 23,1 anos de idade. A idade dos barcos de madeira mostrou concentração entre 21 e 35 anos, sendo que quase a metade deles se encontrava na faixa de 26-30 anos; em média, estavam com 30,9 anos de idade. No total da frota, a concentração ocorreu entre 21 - 35, estando quase a metade dos barcos com 26 - 30 anos; a média geral ficou em 29,6 anos de idade (Tabela II).

Em verdade, a frota camaroneira baseada em portos do estado do Rio de Janeiro tem idade avançada, com pouca renovação. Uma frota com idade avançada torna-se progressivamente mais ineficiente, operando com custo crescente e produtividade decres- 
cente (Paiva, 1986). Isto comprova a baixa capacidade de novos investimentos dos armadores e/ou insuficiente produtividade das pescarias do camarão-rosa.

Em geral, a frota camaroneira baseada em portos do estado do Rio de Janeiro opera durante 8 9 meses por ano, por causa do período do defeso, que normalmente corresponde a três meses (Tabela III). Fora do período de defeso, que sempre cobre os meses de março - abril, mais de $40 \%$ da frota permanece em operação, com maior atividade nos meses de julho outubro (Tabela IV).

Nos anos em estudo, camaroneiros chegaram ao máximo de 270 dias de pesca/ano, mas normalmente ficam com até 210 dias/ano (TabelaV). A variação do tempo anual de operação se reflete nas capturas do camarão-rosa, em razão direta, crescendo com o aumento do tempo, alcançando pouco mais de $3,8 \mathrm{t} /$ ano, quando as operações se situam entre 181 - 210 dias de pesca/ano, isto em termos médios, para o período de cinco anos (1993 - 1997).

No início deste trabalho, afirmamos a ocorrência de tendência decrescente da produção do camarãorosa, nos desembarques anuais em portos do estado do Rio de Janeiro. Isto decorre, fundamentalmente, da queda na produtividade das pescarias (Tabelas $\mathrm{V}$ VI), e não da redução do número de unidades da frota camaroneira. Não temos dados, na literatura disponível, que possam ser comparados com os que agora apresentamos, o que aumenta a originalidade do nosso trabalho.

Por fim, destacamos a importância dos dados aqui mostrados, tendo em vista o planejamento das operações da frota camaroneira fluminense e os estudos/projetos para sua renovação, por que eles possibilitam conseqüentes avaliações de natureza econômica.

\section{CONCLUSÕES}

As conclusões abaixo apresentadas dizem respeito, tão somente, a pescarias do camarão-rosa efetuadas por barcos industriais, baseados em portos do estado do Rio de Janeiro (1993 - 1997).

1. As características mais estáveis dos barcos camaroneiros são as seguintes: barcos de aço - comprimento total, boca moldada, pontal e calados; barcos de madeira - comprimento total, boca moldada e pontal.

2. Os barcos em operação atingiram o máximo de 55 anos de idade, com as médias seguintes: barcos de aço $=23,1$ anos, barcos de madeira $=30,9$ anos e total da frota $=29,6$ anos. A frota camaroneira tem idade avançada, com pouca renovação.

3. Em geral, a frota camaroneira opera durante $8-9$ meses por ano, por causa do período do defeso, que normalmente corresponde a três meses, sempre cobrindo os meses de março e abril. Fora do período do defeso, mais de $40 \%$ da frota camaroneira permanece em operação, com maior atividade nos meses de julho - outubro.

4. Camaroneiros chegaram ao máximo de 270 dias de pesca/ano, mas normalmente não ultrapassam o limite de 210 dias de pesca/ano.

5. A variação do tempo anual de operação dos barcos se reflete nas capturas do camarão-rosa, crescendo com o aumento do tempo. Em termos médios, quando as operações ficaram entre $181-210$ dias de pesca/ano, a produção do camarão-rosa alcançou pouco mais de $3,8 \mathrm{t} /$ ano.

6. Confirmou-se a tendência decrescente da produção do camarão-rosa, nos sucessivos anos estudados, em decorrência da queda na produtividade das pescarias.

\section{REFERÊNCIAS BIBLIOGRÁFICAS}

Costa, H. et al. Uma avaliação da qualidade das águas costeiras do estado do Rio de Janeiro. Fundação de Estudos do Mar, 261 p., Rio de Janeiro, 1998.

Paiva, M. P. Fundamentos da administração pesqueira. Editerra Editorial Ltda., 156 p., Brasília, 1986.

Paiva, M. P. Recursos pesqueiros estuarinos e marinhos do Brasil. Edições UFC, 286 p., Fortaleza, 1997. 